\title{
MARKOV PROCESSES WITH RESTART
}

\author{
KONSTANTIN AVRACHENKOV,${ }^{*}$ Inria Sophia Antipolis \\ ALEXEY PIUNOVSKIY ***** AND \\ YI ZHANG, ${ }^{* * * * * *}$ The University of Liverpool
}

\begin{abstract}
We consider a general homogeneous continuous-time Markov process with restarts. The process is forced to restart from a given distribution at time moments generated by an independent Poisson process. The motivation to study such processes comes from modeling human and animal mobility patterns, restart processes in communication protocols, and from application of restarting random walks in information retrieval. We provide a connection between the transition probability functions of the original Markov process and the modified process with restarts. We give closed-form expressions for the invariant probability measure of the modified process. When the process evolves on the Euclidean space, there is also a closed-form expression for the moments of the modified process. We show that the modified process is always positive Harris recurrent and exponentially ergodic with the index equal to (or greater than) the rate of restarts. Finally, we illustrate the general results by the standard and geometric Brownian motions.

Keywords: Markov process with restart; positive Harris recurrence; exponential ergodicity; standard and geometric Brownian motions
\end{abstract}

2010 Mathematics Subject Classification: Primary 60J25

Secondary 47D07

\section{Introduction}

Many phenomena in nature and technology can be modeled by Markov processes which restart from time to time. Human and animal movements can be modeled by Markov processes that restart from some locations [8], [17]. Facing the congestion of the Internet traffic, the Internet users tend to restart their sessions [10], [13] or a protocol governing the rate of information transmission invokes restart routines (i.e. slow-start routine in the Internet transmission control protocol) [16]. The PageRank algorithm [3] in information retrieval models the behavior of a random surfer, who decides to restart web surfing from time to time. The heat kernel PageRank [4] is a continuous-time analog of the original discrete-time algorithm. The restart policy is also used to speedup the Las Vegas type randomized algorithms [1], [12].

Motivated by the abovementioned phenomena, we study a general homogeneous continuoustime Markov process with restarts. We assume that the process of restarts is modeled by an independent Poisson process. In Section 2 we derive a formula which makes a connection between the transition probability functions of the original Markov process and the Markov process modified by restarts. As a corollary, we obtain a closed-form expression for the invariant

Received 13 July 2012; revision received 19 February 2013.

* Postal address: Inria Sophia Antipolis, 2004 Route des Lucioles, Sophia Antipolis, 06902, France.

Email address: k.avrachenkov@sophia.inria.fr

** Postal address: Department of Mathematical Sciences, University of Liverpool, M\&O Building, L69 7ZL, UK.

*** Email address: piunov@ liverpool.ac.uk

**** Email address: yi.zhang@liverpool.ac.uk 
probability measure of the modified process. We also show that the modified process is always positive Harris recurrent and exponentially ergodic with the index equal to (or greater than) the rate of restarts. In Section 3 we provide bounds for the moments of the modified process and investigate the limiting behavior of the modified process when the rate of restarts goes to 0 . We conclude the paper with Section 4 where we consider the restart modifications of the two classical Markov processes: the standard Brownian motion with drift and the geometric Brownian motion. It is very curious to observe that in the case of the geometric Brownian motion the number of finite moments of the modified process depends on the rate of restarts. Thus, even a small change in the value of the restart rate can lead to dramatic changes of the properties of the geometric Brownian motion with restarts.

\section{Main results}

In accordance with [11], we consider a homogeneous continuous-time Markov process $\{X(t): t \in[0, \infty)\}$ in a Borel space $(E, \mathcal{E})$ characterized by the initial distribution $\gamma(\cdot)$, and the (honest) transition function $P(t, x, \mathrm{~d} y)$, satisfying the following properties:

1. $P(t, x, \cdot)$ is a probability measure on $\mathcal{E}$, i.e. the transition function is honest;

2. $P(0, x, \Gamma)=\mathbf{1}\{x \in \Gamma\}$;

3. for each fixed $\Gamma \in \mathcal{E}, t \in[0, \infty), P(t, x, \Gamma)$ is a jointly measurable function with respect to $(t, x) \in[0, \infty) \times E$; and

4. the Chapman-Kolmogorov equation holds:

$$
P(t+s, x, \Gamma)=\int_{E} P(s, y, \Gamma) P(t, x, \mathrm{~d} y) .
$$

Throughout this paper, all the processes are from the same probability space $(\Omega, \mathcal{F}, \mathbb{P})$. We further use the notation $\mathbb{P}_{x}, \mathbb{E}_{x}$, and $\operatorname{var}_{x}$ when the initial state of the concerned process is $x \in E$.

The goal of the present work is to analyse a modification of the Markov process $\{X(t): t \in$ $[0, \infty)\}$ introduced in the above by forcing the process to restart with a given restart distribution $v(\Gamma)$ after an exponentially distributed random time, i.e. the process counting restarts represents a standard Poisson process with rate, say, $\lambda>0$, independent of $\{X(t): t \in[0, \infty)\}$. In the following theorem we characterize the transition function of the modified Markov process, which is denoted as $\{\tilde{X}(t): t \in[0, \infty)\}$.

Theorem 2.1. Let $P(t, x, \Gamma)$ be the transition function of the Markov process $\{X(t): t \in$ $[0, \infty)\}$ in a Borel space $(E, \mathcal{E})$. Then the modified Markov process $\{\tilde{X}(t), t \in[0, \infty)\}$ that restarts from a distribution $v$ after (independent) exponentially distributed random times with mean $1 / \lambda$ has the following transition function:

$$
\tilde{P}_{v}(t, x, \Gamma)=\mathrm{e}^{-\lambda t} P(t, x, \Gamma)+\int_{E} \int_{0}^{t} \lambda \mathrm{e}^{-\lambda s} P(s, y, \Gamma) \mathrm{d} s v(\mathrm{~d} y) .
$$

Proof. Denote by $N(t)$ the number of restarts up to time $t$ and by $S$ the time elapsed since the time moment of the last restart before $t$. Then we have

$$
\begin{aligned}
\tilde{P}_{v}(t, x, \Gamma)= & \mathbb{P}[\{\tilde{X}(t) \in \Gamma\} \cap\{N(t)=0\} \mid \tilde{X}(0)=x] \\
& +\mathbb{P}[\{\tilde{X}(t) \in \Gamma\} \cap\{N(t)>0\} \mid \tilde{X}(0)=x] .
\end{aligned}
$$


The first term on the left-hand side can be written as

$$
\mathbb{P}[\{\tilde{X}(t) \in \Gamma\} \cap\{N(t)=0\} \mid \tilde{X}(0)=x]=\mathrm{e}^{-\lambda t} P(t, x, \Gamma) .
$$

Let us now calculate the second term on the right-hand side of (2.2). Note that this term can be written as

$$
\mathbb{P}[\{\tilde{X}(t) \in \Gamma\} \cap\{N(t)>0\} \mid \tilde{X}(0)=x]=\int_{E} \int_{0}^{t} P(s, y, \Gamma) \mathrm{d} F(s) v(\mathrm{~d} y),
$$

where $F(s)=\mathbb{P}[S \leq s, N(t)>0]$. Let $T_{k}$ denote the $k$ th restart moment from time 0 . If $N(t)=n>0$ then the restart times $T_{1}, \ldots, T_{n}$ have the same distribution as the order statistics corresponding to $n$ independent random variables uniformly distributed on the interval $(0, t)$ [15], and, therefore,

$$
\mathbb{P}\left[T_{n} \leq \tau \mid N(t)=n\right]=\left(\frac{\tau}{t}\right)^{n}, \quad \tau \in[0, t], n=1,2, \ldots
$$

Then,

$$
\mathbb{P}[S \leq s \mid N(t)=n]=\mathbb{P}\left[T_{n}>t-s \mid N(t)=n\right]=1-\left(\frac{t-s}{t}\right)^{n}, \quad n=1,2, \ldots,
$$

and, consequently,

$$
F(s)=\sum_{n=1}^{\infty} \frac{(\lambda t)^{n}}{n !} \mathrm{e}^{-\lambda t}\left[1-\left(\frac{t-s}{t}\right)^{n}\right]=\mathrm{e}^{-\lambda t}\left[\mathrm{e}^{\lambda t}-\mathrm{e}^{\lambda(t-s)}\right]=1-\mathrm{e}^{-\lambda s} .
$$

Hence,

$$
\mathbb{P}[\{\tilde{X}(t) \in \Gamma\} \cap\{N(t)>0\} \mid \tilde{X}(0)=x]=\int_{E} \int_{0}^{t} \lambda \mathrm{e}^{-\lambda s} P(s, y, \Gamma) \mathrm{d} s v(\mathrm{~d} y),
$$

and (2.1) follows.

Remark 2.1. Our proof is based on conditioning upon the elapsed time since the last restart. If we condition upon the time until the first restart then we obtain the following equation:

$$
\tilde{P}_{v}(t, x, \Gamma)=\mathrm{e}^{-\lambda t} P(t, x, \Gamma)+\int_{E} \int_{0}^{t} \lambda \mathrm{e}^{-\lambda(t-s)} \tilde{P}_{\nu}(s, y, \Gamma) \mathrm{d} s v(\mathrm{~d} y) .
$$

Corollary 2.1. The measure

$$
q_{\nu}(\Gamma)=\int_{E} \int_{0}^{\infty} \lambda \mathrm{e}^{-\lambda s} P(s, y, \Gamma) \mathrm{d} s v(\mathrm{~d} y)
$$

is an invariant probability measure for $\tilde{P}_{\nu}(t, x, \Gamma)$.

Proof. Since $P(s, y, \cdot)$ is a probability measure for all $s$ and $y, q_{v}(\cdot)$ is a probability measure as well. Let us show that it is indeed an invariant measure. It is enough to show this for 
$v(\mathrm{~d} y)=\mathbf{1}\{w \in \mathrm{d} y\}$, where $w \in E$ is fixed. In this case we also denote $q_{v}$ by $q_{w}$ and $\tilde{P}_{v}$ by $\tilde{P}_{w}$ for brevity. By Theorem 2.1 we obtain

$$
\begin{aligned}
\int_{E} q_{w}(\mathrm{~d} z) \tilde{P}_{w}(t, z, \Gamma) & =\int_{E} q_{w}(\mathrm{~d} z)\left[\mathrm{e}^{-\lambda t} P(t, z, \Gamma)+\int_{0}^{t} \lambda \mathrm{e}^{-\lambda s} P(s, w, \Gamma) \mathrm{d} s\right] \\
& =\mathrm{e}^{-\lambda t} \int_{E} q_{w}(\mathrm{~d} z) P(t, z, \Gamma)+\int_{0}^{t} \lambda \mathrm{e}^{-\lambda s} P(s, w, \Gamma) \mathrm{d} s .
\end{aligned}
$$

Substituting into the above equation

$$
q_{w}(\Gamma)=\int_{0}^{\infty} \lambda \mathrm{e}^{-\lambda s} P(s, w, \Gamma) \mathrm{d} s,
$$

and using the Chapman-Kolmogorov equation yield

$$
\begin{aligned}
\int_{E} q_{w}(\mathrm{~d} z) \tilde{P}_{w}(t, z, \Gamma) & =\mathrm{e}^{-\lambda t} \int_{0}^{\infty} \lambda \mathrm{e}^{-\lambda s} P(s+t, w, \Gamma) \mathrm{d} s+\int_{0}^{t} \lambda \mathrm{e}^{-\lambda s} P(s, w, \Gamma) \mathrm{d} s \\
& =\int_{t}^{\infty} \lambda \mathrm{e}^{-\lambda s^{\prime}} P\left(s^{\prime}, w, \Gamma\right) \mathrm{d} s^{\prime}+\int_{0}^{t} \lambda \mathrm{e}^{-\lambda s} P(s, w, \Gamma) \mathrm{d} s \\
& =\int_{0}^{\infty} \lambda \mathrm{e}^{-\lambda s} P(s, w, \Gamma) \mathrm{d} s \\
& =q_{w}(\Gamma),
\end{aligned}
$$

which concludes the proof of the corollary.

Remark 2.2. We note that (2.3) can also be rewritten as

$$
q_{v}(\Gamma)=\int_{E} \lambda R(y, \Gamma) v(\mathrm{~d} y),
$$

where $R(y, \Gamma)=\int_{0}^{\infty} \mathrm{e}^{-\lambda s} P(s, y, \Gamma) \mathrm{d} s$ is the resolvent operator.

Now we are ready to prove that the modified process is positive Harris recurrent and exponentially ergodic with index $\lambda$. Before this, we remind the reader of some definitions.

Recall that a homogeneous continuous-time Markov process $\{\tilde{X}(t), t \in[0, \infty)\}$ is called Harris recurrent if there exists a nontrivial $\sigma$-finite (recurrence) measure $\mu$ on $\mathscr{E}$ such that, for each $x \in E$,

$$
\tau_{\Gamma}:=\inf \{t \geq 0: \tilde{X}(t) \in \Gamma\}<\infty \quad P_{x} \text {-almost surely, }
$$

whenever $\mu(\Gamma)>0$; see [7] and [14]. If a Harris recurrent process admits an invariant probability measure, it must be unique, and the process is further called positive Harris recurrent; see [7] and [14].

A homogeneous continuous-time Markov process $\{\tilde{X}(t), t \in[0, \infty)\}$ is called exponentially ergodic with index $\alpha$ if there exist a probability measure $\mu(\cdot)$ on $\mathcal{E}$, a finite-valued function $M(\cdot)$ on $\mathrm{E}$, and a constant $\alpha>0$ satisfying $\|\tilde{P}(t, x, \cdot)-\mu(\cdot)\|_{\mathrm{TV}} \leq M(x) \mathrm{e}^{-\alpha t}$ for every $x \in E$, where $\|\cdot\|_{\mathrm{TV}}$ denotes the total variation norm; see [5].

Theorem 2.2. The modified Markov process $\{\tilde{X}(t), t \in[0, \infty)\}$ is positive Harris recurrent and exponentially ergodic with index equal to (or greater than) the rate of restarts $\lambda$, and the following inequality holds:

$$
\left|q_{v}(\Gamma)-\tilde{P}_{\nu}(t, x, \Gamma)\right| \leq \mathrm{e}^{-\lambda t} \text { for all } x \in \text { Eand all } \Gamma \in \mathcal{E} .
$$


Proof. The modified process $\{\tilde{X}(t), t \in[0, \infty)\}$ under consideration is Harris recurrent with the recurrence measure given by the restart distribution $\mu(\cdot)=v(\cdot)$. From Corollary 2.1 we conclude that $\{\tilde{X}(t), t \in[0, \infty)\}$ is positive Harris recurrent with the unique invariant probability measure given by (2.3). Let us now prove (2.4). We see that

$$
\begin{aligned}
\left|q_{v}(\Gamma)-\tilde{P}_{\nu}(t, x, \Gamma)\right| & =\left|\int_{E} \int_{t}^{\infty} \lambda \mathrm{e}^{-\lambda s} P(s, y, \Gamma) \mathrm{d} s v(\mathrm{~d} y)-\mathrm{e}^{-\lambda t} P(t, x, \Gamma)\right| \\
& =\left|\int_{E} \int_{t}^{\infty} \lambda \mathrm{e}^{-\lambda s} P(s, y, \Gamma) \mathrm{d} s v(\mathrm{~d} y)-\int_{t}^{\infty} \lambda \mathrm{e}^{-\lambda s} P(t, x, \Gamma) \mathrm{d} s\right| \\
& =\left|\int_{t}^{\infty} \lambda \mathrm{e}^{-\lambda s}\left[\int_{E} P(s, y, \Gamma) v(\mathrm{~d} y)-P(t, x, \Gamma)\right] \mathrm{d} s\right| \\
& \leq \int_{t}^{\infty} \lambda \mathrm{e}^{-\lambda s}\left|\int_{E} P(s, y, \Gamma) v(\mathrm{~d} y)-P(t, x, \Gamma)\right| \mathrm{d} s \\
& \leq \mathrm{e}^{-\lambda t} .
\end{aligned}
$$

Since $\left\|\tilde{P}(t, x, \cdot)-q_{v}(\cdot)\right\|_{\mathrm{TV}}=2 \sup _{\Gamma \in \mathcal{E}}\left|\tilde{P}(t, x, \Gamma)-q_{v}(\Gamma)\right|[9$, Appendix $]$, it follows that $\{\tilde{X}(t), t \in[0, \infty)\}$ is exponentially ergodic with index $\lambda$ and $M(x)=2$.

\section{Moments and limits}

In this section we let the initial distribution of the original process be the Dirac measure concentrated at $x \in E$, where $E=\mathbb{R}^{n}$. Then we write $\{\tilde{X}(t): t \geq 0\}$ as

$$
\left\{\left(\tilde{X}_{1}(t), \ldots, \tilde{X}_{n}(t)\right): t \geq 0\right\} .
$$

Similar notation is introduced for the process $\{X(t): t \geq 0\}$. Now consider the $i$ th component process $\left\{\tilde{X}_{i}(t), t \geq 0\right\}$. From (2.1) we can obtain an expression for the moments

$$
\mathbb{E}_{x}\left[\tilde{X}_{i}^{k}(t)\right]=\mathrm{e}^{-\lambda t} \mathbb{E}_{x}\left[X_{i}^{k}(t)\right]+\int_{E} \int_{0}^{t} \lambda \mathrm{e}^{-\lambda s} \mathbb{E}_{y}\left[X_{i}^{k}(s)\right] \mathrm{d} s v(\mathrm{~d} y),
$$

where, here and below, we assume that the involved interchange of the order of integrals is legal, which is the case, for example, when

$$
\int_{E} \int_{0}^{t} \lambda \mathrm{e}^{-\lambda s} E_{y}\left[\left|X_{i}^{k}(s)\right|\right] \mathrm{d} s v(\mathrm{~d} y)<\infty .
$$

In turn, (3.1) helps to establish the following bound.

Proposition 3.1. Let the kth moment of the original process be exponentially bounded from the above in time, i.e.

$$
\mathbb{E}_{x}\left[X_{i}^{k}(t)\right] \leq c_{k, i}(x) \mathrm{e}^{\eta_{k, i} t}
$$

where $c_{k, i}(\cdot)$ is a measurable $v$-integrable function, and $\eta_{k, i}<\lambda$ is a constant. Then

$$
\limsup _{t \rightarrow \infty} \mathbb{E}_{x}\left[\tilde{X}_{i}^{k}(t)\right] \leq \frac{\bar{c}_{k, i} \lambda}{\lambda-\eta_{k, i}},
$$

where $\bar{c}_{k, i}=\int_{E} c_{k, i}(y) v(\mathrm{~d} y)$. If the kth moment of the original process is uniformly bounded from the above (i.e. $\mathbb{E}_{x}\left[X_{i}^{k}(t)\right] \leq c_{k, i} \in(-\infty, \infty)$ ), so is the kth moment of the modified process by the same bound (i.e. $\left.E_{x}\left[\tilde{X}_{i}^{k}(t)\right] \leq c_{k, i}\right)$. 
Proof. Equation (3.1) yields

$$
\begin{aligned}
\mathbb{E}_{x}\left[\tilde{X}_{i}^{k}(t)\right] & \leq \mathrm{e}^{-\lambda t} c_{k, i}(x) \mathrm{e}^{\eta_{k, i} t}+\int_{E} \int_{0}^{t} \lambda \mathrm{e}^{-\lambda s} c_{k, i}(y) \mathrm{e}^{\eta_{k, i} s} \mathrm{~d} s v(\mathrm{~d} y) \\
& =\mathrm{e}^{-\left(\lambda-\eta_{k, i}\right) t} c_{k, i}(x)+\frac{\lambda}{\lambda-\eta_{k, i}}\left(1-\mathrm{e}^{-\left(\lambda-\eta_{k, i}\right) t}\right) \int_{E} c_{k, i}(y) v(\mathrm{~d} y),
\end{aligned}
$$

which implies the first statement of the proposition. The second statement of the proposition also follows from (3.1).

Now let us investigate what happens when the parameter $\lambda$ goes to 0 . Since $\int_{E} P(s, y, \Gamma) v(\mathrm{~d} y)$ is a bounded measurable function with respect to $s$, according to [6], we can conclude that the limiting discounting is equivalent to the time averaging. This fact and Theorem 1 of [7] lead to the following statement.

Theorem 3.1. The existence of the limit $\lim _{\lambda \rightarrow 0} q_{\nu}(\Gamma)$ is equivalent to the existence of the limit $\lim _{T \rightarrow \infty}(1 / T) \int_{0}^{T} \int_{E} P(s, y, \Gamma) v(\mathrm{~d} y) \mathrm{d} s$. If these limits exist, they are equal. In particular, if the original process $\{X(t): t \in[0, \infty)\}$ is positive Harris recurrent, the limit $\lim _{\lambda \rightarrow 0} q_{v}(\Gamma)$ exists and is equal to the invariant probability measure of the original process.

Remark 3.1. There could be cases when the $\operatorname{limit}_{\lambda \rightarrow 0} q_{v}(\cdot)$ exists for any probability distribution $v(\cdot)$. However, similarly to the case of singularly perturbed Markov processes [2], the original process might not be ergodic.

\section{Examples}

Let us illustrate the general results with two examples.

\subsection{Brownian motion with drift}

As the first example, let us consider the Brownian motion with drift $\mu$ and variance coefficient $\sigma^{2}$ on the real line $E=\mathbb{R}$ (see, e.g. [15]). The initial distribution of the original process is the Dirac measure concentrated at $x \in E$. It can be described in stochastic differential notation as

$$
\mathrm{d} X(t)=\mu \mathrm{d} t+\sigma \mathrm{d} W(t),
$$

where $W(t)$ is the standard Wiener process. The probability density function of the Brownian process has a closed form:

$$
p(t, 0, z)=\frac{1}{\sqrt{2 \pi \sigma^{2} t}} \exp \left(-\frac{(z-\mu t)^{2}}{2 \sigma^{2} t}\right) .
$$

Here we assume that the process starts from 0 . We observe that $p(t, 0, z)$ does not converge to a proper probability density as $t$ goes to $\infty$. If the modified process also restarts from 0 , by (2.1), we have

$$
\begin{aligned}
\tilde{p}(t, 0, z)= & \exp (-\lambda t) \frac{1}{\sqrt{2 \pi \sigma^{2} t}} \exp \left(-\frac{(z-\mu t)^{2}}{2 \sigma^{2} t}\right) \\
& +\lambda \int_{0}^{t} \exp (-\lambda s) \frac{1}{\sqrt{2 \pi \sigma^{2} s}} \exp \left(-\frac{(z-\mu s)^{2}}{2 \sigma^{2} s}\right) \mathrm{d} s,
\end{aligned}
$$

which has the well-defined limiting probability density function

$$
q_{0}(z)=\lambda \int_{0}^{\infty} \exp (-\lambda s) \frac{1}{\sqrt{2 \pi \sigma^{2} s}} \exp \left(-\frac{(z-\mu s)^{2}}{2 \sigma^{2} s}\right) \mathrm{d} s .
$$


Let us now consider that the original process starts with an arbitrarily fixed $x \in E$ and restarts according to the distribution $v(\mathrm{~d} y)$, which admits a finite second moment. Then we may calculate the first moment of the modified process by (3.1) with $\mathbb{E}_{x}[X(t)]=x+\mu t$ :

$$
\begin{aligned}
\mathbb{E}_{x}[\tilde{X}(t)] & =\mathrm{e}^{-\lambda t}(x+\mu t)+\int_{E} \int_{0}^{t} \lambda \mathrm{e}^{-\lambda s}(y+\mu s) \mathrm{d} s v(\mathrm{~d} y) \\
& =\mathrm{e}^{-\lambda t}(x+\mu t)+\left[1-\mathrm{e}^{-\lambda t}\right] \int_{E} y v(\mathrm{~d} y)+\left[1-(1+\lambda t) \mathrm{e}^{-\lambda t}\right] \frac{\mu}{\lambda} .
\end{aligned}
$$

Thus,

$$
\mathbb{E}_{x}[\tilde{X}(t)] \rightarrow \int_{E} y v(\mathrm{~d} y)+\frac{\mu}{\lambda} \quad \text { as } t \rightarrow \infty .
$$

Similarly, a direct calculation gives

$$
\mathbb{E}_{x}\left[\tilde{X}^{2}(t)\right] \rightarrow \frac{\sigma^{2}}{\lambda}+\frac{2 \mu^{2}}{\lambda^{2}}+\int_{E}\left(\frac{2 \mu y}{\lambda}+y^{2}\right) v(\mathrm{~d} y) \text { as } t \rightarrow \infty,
$$

and, thus,

$$
\operatorname{var}_{x}\left[\tilde{X}^{2}(t)\right] \rightarrow \int_{E} y^{2} v(\mathrm{~d} y)-\left(\int_{E} y v(\mathrm{~d} y)\right)^{2}+\frac{\sigma^{2}}{\lambda}+\frac{\mu^{2}}{\lambda^{2}} \quad \text { as } t \rightarrow \infty .
$$

\subsection{Geometric Brownian motion}

As the second example, we consider the geometric Brownian motion (see, e.g. [15]), so we take $E=[0, \infty)$. It can be described by the stochastic differential equation (with the initial condition $\mathbb{P}[X(0) \in \mathrm{d} y]=\mathbf{1}\{x \in \mathrm{d} y\}$, where $x \in E$ is fixed)

$$
\mathrm{d} X(t)=\mu X(t) \mathrm{d} t+\sigma X(t) \mathrm{d} W(t)
$$

where $W(t)$ is the standard Wiener process. The probability density function of the geometric Brownian motion also has a closed form, i.e.

$$
p(t, x, z)=\frac{1}{\sqrt{2 \pi}} \frac{1}{z \sigma \sqrt{t}} \exp \left(-\frac{\left(\ln (z)-\ln (x)-\left(\mu-\sigma^{2} / 2\right) t\right)^{2}}{2 \sigma^{2} t}\right),
$$

which defines a log-normal distribution with mean

$$
\mathbb{E}_{x}[X(t)]=x \mathrm{e}^{\mu t}
$$

and variance

$$
\operatorname{var}_{x}[X(t)]=x^{2} \mathrm{e}^{2 \mu t}\left(\mathrm{e}^{\sigma^{2} t}-1\right) .
$$

Indeed, for any $k=1,2, \ldots$, it holds for this log-normal distribution that

$$
\mathbb{E}_{x}\left[X^{k}(t)\right]=x^{k} \exp \left(k\left(\mu-\frac{\sigma^{2}}{2}\right) t+\frac{k^{2} \sigma^{2} t}{2}\right) .
$$


Next, for any fixed $k=1,2, \ldots$, we assume that $\lambda-k\left(\mu-\sigma^{2} / 2\right)-\frac{1}{2} k^{2} \sigma^{2} \neq 0$, and $v(\mathrm{~d} y)$ has a finite $k$ th moment. By using (3.1), the $k$ th moment of the modified process is given by

$$
\begin{aligned}
\mathbb{E}_{x}\left[\tilde{X}^{k}(t)\right]= & \mathrm{e}^{-\lambda t} x^{k} \exp \left(k\left(\mu-\frac{\sigma^{2}}{2}\right) t+\frac{1}{2} k^{2} \sigma^{2} t\right) \\
& +\int_{E} \int_{0}^{t} \lambda \mathrm{e}^{-\lambda s} y^{k} \exp \left(k\left(\mu-\frac{\sigma^{2}}{2}\right) s+\frac{1}{2} k^{2} \sigma^{2} s\right) \mathrm{d} s v(\mathrm{~d} y) \\
= & x^{k} \exp \left(-t\left(\lambda-k\left(\mu-\frac{\sigma^{2}}{2}\right)-\frac{1}{2} k^{2} \sigma^{2}\right)\right) \\
& +\int_{E} \lambda y^{k} \int_{0}^{t} \exp \left(-s\left(\lambda-k\left(\mu-\frac{\sigma^{2}}{2}\right)-\frac{1}{2} k^{2} \sigma^{2}\right)\right) \mathrm{d} s v(\mathrm{~d} y) \\
= & x^{k} \exp \left(-t\left(\lambda-k\left(\mu-\frac{\sigma^{2}}{2}\right)-\frac{1}{2} k^{2} \sigma^{2}\right)\right) \\
& +\lambda \frac{1-\exp \left(-t\left(\lambda-k\left(\mu-\sigma^{2} / 2\right)-k^{2} \sigma^{2} / 2\right)\right)}{\lambda-k\left(\mu-\sigma^{2} / 2\right)-k^{2} \sigma^{2} / 2} \int_{E} y^{k} v(\mathrm{~d} y) .
\end{aligned}
$$

Thus, when $\lambda>k\left(\mu-\sigma^{2} / 2\right)+\frac{1}{2} k^{2} \sigma^{2}>0$, we have

$$
\mathbb{E}_{x}\left[\tilde{X}^{k}(t)\right] \rightarrow \frac{\lambda}{\lambda-k\left(\mu-\sigma^{2} / 2\right)-k^{2} \sigma^{2} / 2} \int_{E} y^{k} v(\mathrm{~d} y) \quad \text { as } t \rightarrow \infty .
$$

Let us provide some intuition why in the case of the geometric Brownian motion with restart the number of moments that converge to a finite limit depends on the restart rate. Consider a piecewise-deterministic process driven by a Poisson process with rate $\lambda$, whose deterministic component between two consecutive restart moments is described by $x_{0} \exp (\mu u)$, where $u$ is the time after the last restart (compare with (4.1)). This process can also be viewed as a restart process whose original process has the transition function

$$
P(t, x, \Gamma)=\mathbf{1}\left\{x \mathrm{e}^{\mu t} \in \Gamma\right\} .
$$

Note that $\int_{0}^{\infty} z P(t, x, \mathrm{~d} z)=x \exp (\mu t)$, which is equal to (4.1). The substitution of the above transition function into (2.3) yields the Pareto steady-state distribution

$$
P(\tilde{X}(t) \geq x)=\left(\frac{x_{0}}{x}\right)^{\lambda / \mu},
$$

which can have only a finite number of finite moments.

\section{Acknowledgement}

This work was partially supported by the RCMM (Department of Mathematical Sciences, University of Liverpool), under grant 1412-08PIU, and by the European Commission within the framework of the CONGAS project FP7-ICT-2011-8-317672.

\section{References}

[1] Alt, H., et al. (1996). A method for obtaining randomized algorithms with small tail probabilities. Algorithmica 16, 543-547. 
[2] Avrachenkov, K. E., Filar, J. and Haviv, M. (2002). Singular perturbations of Markov chains and decision processes. In Handbook of Markov Decision Processes, eds E. A. Feinberg and A. Shwartz, Kluwer, Boston, MA, pp. 113-150.

[3] Brin, S. And Page, L. (1998). The anatomy of a large-scale hypertextual Web search engine. Comput. Networks ISDN Systems 30, 107-117.

[4] Chung, F. (2007). The heat kernel as the pagerank of a graph. Proc. Nat. Acad. Sci. 104, 19735-19740.

[5] Down, D., Meyn, S. P. and Tweedie, R. L. (1995). Exponential and uniform ergodicity of Markov processes. Ann. Prob. 23, 1671-1691.

[6] Feller, W. (1971). An Introduction to Probability Theory and Its Applications, Vol. II, 2nd edn. John Wiley, New York.

[7] Glynn, P. W. (1994). Some topics in regenerative steady-state simulation. Acta Appl. Math. 34, 225-236.

[8] González, M. C., Hidalgo, C. A. and Barabási, A.-L. (2008). Understanding individual human mobility patterns. Nature 453, 779-782.

[9] Hernández-Lerma, O. and Lasserre, J.-B. (1996). Discrete-time Markov Control Processes, Springer, New York.

[10] Krishnamurthy, B. And Rexford, J. (2001). Web Protocols and Practice: HTTP/1.1, Networking Protocols, Caching, and Traffic Measurement. Addison Wesley.

[11] Kuznetsov, S. E. (1980). Any Markov process in a Borel space has a transition function. Theory Prob. Appl. 25, 384-388.

[12] Luby, M., Sinclair, A. And Zuckerman, D. (1993). Optimal speedup of Las Vegas algorithms. Inf. Process. Lett. 47, 173-180.

[13] Maurer, S. M. And Huberman, B. A. (2001). Restart strategies and Internet congestion. J. Econom. Dynamics Control 25, 641-654.

[14] Meyn, S. P. And Tweedie, R. L. (1993). Stability of Markov processes III. Forster-Lyapunov criteria for continuous-time processes. Adv. Appl. Prob. 25, 518-548.

[15] Ross, S. M. (1996). Stochastic Processes, 2nd edn. John Wiley, New York.

[16] Stevens, W. R. (1994). TCP/IP Illustrated, Volume 1: The Protocols. Addison Wesley.

[17] Walsh, P. D., Boyer, D. and Crofoot, M. C. (2010). Monkey and cell-phone-user mobilities scale similarly. Nature Phys. 6, 929-930. 